\title{
Are Colours Visually Complex?
}

\section{Pär Sundström}

\section{Introduction}

Squarehood is a visually complex property in the following sense. To be square is to have certain parts or aspects - four lines of equal length connected at right angles - that are visually accessible and none of which is identical with squarehood. And to see something as being square is to see it as having these parts or aspects.

It is often supposed that colours are not thus visually complex. For example, I think we can take Locke to express this view when he mentions colours among the

simple Ideas; which being each in itself uncompounded, contains in it nothing but one uniform Appearance, or Conception in the mind, and is not distinguishable into different Ideas. (Locke 1975 [1689]: sect. 2.2.1)

Similarly, Hume, discussing blue, green, scarlet and "particular sounds, and tastes and smells", says that "their very nature ... excludes all composition" (Hume 1978 [1739]: 637). ${ }^{1}$

Perhaps this view of colour is ultimately correct. However, I am not sure it is correct. I think we should take seriously the hypothesis that colours - all colours - are visually complex in the above explained sense. This paper tries to explain why I think we should take this seriously.

Section 2 presents a case that almost all shades of colour are visually complex. I will not fully articulate the case but I hope to say enough to convey that it is strong. Section 3 presents a more tentative case that the remaining colours are visually complex as well.

\section{Almost All Shades of Colour Are Visually Complex}

There is, I believe, a strong case to be made that almost all shades of colour are visually complex. Consider a contemporary phenomeno-

\footnotetext{
${ }^{1}$ See Mizrahi (2009) for another, recent endorsement of this kind of view.
} 
logical colour model, like the Natural Color System, NCS. ${ }^{1}$ This system, or model, takes there to be six "elementary attributes", ${ }^{2}$ blackness, whiteness, redness, yellowness, greenness and blueness, and it takes each shade of colour to be composed in a quantifiable way by 1-4 of these attributes. For example, the greyish-orange shade 3020-Y50R is composed by blackness, whiteness, redness and yellowness. The initial 30 in the notation says that this shade is composed by $30 \%$ blackness. The ensuing 20 says that its proportion of chromatic attributes (redness, yellowness, greenness or blueness) to achromatic attributes (blackness or whiteness) is 20:80. The Y50R says that its chromatic component is composed by equal proportions of yellowness and redness. One can derive from this that the shade in question is composed by the following shades in the following proportions: $10 \%$ redness, $10 \%$ yellowness, $30 \%$ blackness, and 50\% whiteness. ${ }^{3}$

To judge this kind of model fairly it is important to keep it clearly apart from other models of colour, and two in particular. First, there are additive colour models that serve to systematise which perceived colours will be projected on a screen by various mixtures of monochromatic light. Additive colour models tell us, among other things, that we can project an image of yellow on a screen by blending, in the right proportions, monochromatic light that by itself would project an image of green on a screen and monochromatic light that by itself would project an image of red. Second, there are subtractive colour models that serve to systematise what colours result from mixtures of various pigments. Subtractive colour models tell us, among other things, that a colour printer will print green if it mixes certain proportions of yellow and cyan. It is clear that additive and subtractive colour models are designed for different purposes, make different and supplementary claims and that they systematise colours in nonequivalent ways without being in conflict with one another. It should also be clear that each of them differs in the

\footnotetext{
${ }^{1}$ For expositions see Hård and Sivik (1981), Hård and Svedmyr (1995) and Hård et al. (1996). This kind of model is usually traced to Hering (1964 [1920]), but traces can be discerned earlier in history; see Hård and Svedmyr (1995:39-40) and Pridmore (2006).

${ }^{2}$ Hård et al. (1996: 189).

${ }^{3}$ See Hård and Sivik (1981), Hård and Svedmyr (1995: chapter 2 and pages 138-9) and Hård et al. (1996: part 1).
} 
same ways from the kind of phenomenological colour model that the Natural Color System represents. The latter kind of model serves to systematise how colour visually appear. It tells us about the visual characteristics of 3020-Y50R and other shades. But it makes no claim about which colour pigment mixtures or monochromatic light mixtures will produce these shades, and it is not in conflict with additive or subtractive models even when these systematise colours in ways that do not map onto the phenomenological one. For example, the claim that greenness is a phenomenologically "elementary attribute" is compatible with the claim that one can produce green by certain mixtures of pigments. ${ }^{1}$

To my eyes and mind, the Natural Color System is natural. It seems to me that 3020-Y50R is visually a mixture of blackness, whiteness, redness and yellowness. I gather that I share this sense with many others who are familiar with the model. For example, Hård and Sivik report that, "people without any previous knowledge of colour assessment, other than with common color names, understand and rapidly acquire the NCS method of describing colours - less than 15 minutes is generally required" (Hård and Sivik 1981:137). Moreover, there is reportedly a high degree of agreement between different subjects' specific assessments about how shades of colour are phenomenologically composed. ${ }^{2}$ The system is also widely adopted by professionals in, e.g., architecture, design and painting (see Hård and Svedmyr 1995: chapter 3 - and many local paint stores).

This makes for a strong case, I think, that almost all shades of colour are visually complex in the present sense. For example, 3020-Y50R has multiple component parts or aspects - blackness, whiteness, redness and yellowness - that are visually accessible and none of which is identical with that shade, and to see something as 3020-Y50R is to see it as composed by these attributes in the relevant proportions. Please note:

\footnotetext{
${ }^{1}$ For a bit more on these three types of model and their differences, see Sundström (2008: sect. 4.5) and Byrne and Hilbert (2008: sect. 2).

${ }^{2}$ Interestingly, the agreement reportedly holds even between (a) subjects who make the estimates with the aid of samples of the elementary colours (the shades of colour that are composed by exactly one of the elementary colour attributes) and (b) subjects who make the estimates without such samples, drawing only on their own "inner" understanding of the elementary colours. For details, see Hård and Svedmyr (1995: 67-9) and Hård et al. (1996: 185-7).
} 
I claim that the case is strong, not that it is conclusive. We may well be quite suggestible when it comes to perceptual and introspective reports. ${ }^{1}$ And, as far as I can tell, the test subjects that have been involved in the research and development behind the Natural Color System have had the system suggested to them. Their reports - and mine - may well be tainted by these background suggestions. Nonetheless it seems to me that these reports - in particular, the agreement between them and the related widespread use of the model among professionals in architecture, design and painting - provides strong support for the claim that almost all colours are visually complex. ${ }^{2}$

${ }^{1}$ The "imageless thought controversy" of the early $20^{\text {th }}$ century comes to mind; for an overview see Thomas (2011: sect. 3.2).

${ }^{2}$ As I announced, I do not here fully articulate the case that almost all colours are visually complex. To do so one should address at least two counter-proposals. The first is that, while the Natural Colour System provides a natural ordering of shades of colour, colours do not have this ordering because they are composed by elementary colour attributes in different proportions. One can perhaps trace this kind of proposal to Hume. Hume claims that, "Blue and green are different simple ideas, but are more resembling than blue and scarlet: tho' their perfect simplicity excludes all possibility of separation or distinction. 'Tis the same with particular sounds, and tastes and smells. These admit of infinite resemblances upon the general appearance and comparison, without having any common circumstance the same" (Hume 1978 [1739]: 637). One may perhaps take this to suggest that, e.g., shades of orange bear some natural resemblance to one another but that this resemblance is not rooted in their "common circumstances" redness and yellowness. The second counterproposal to what I have said and that a full defence should take into account is that the Natural Colour System does not even provide a natural ordering of colours. For example, Mizrahi, defending a "conventionalist approach to colour categorization" claims that "the fact that orange is steadily said to be both reddish and yellowish [is] not rooted in the phenomenology of colour experience" (Mizrahi 2009: sect. 4); quoted from the online version, which has the word "in" in place of the above bracketed "is"). Similarly, Saunders and van Brakel claim that the categorisation of chromatic colour in terms of four primitive hues is "rhetorical" (Saunders and van Brakel 1997:173), by which I believe they mean that it is not grounded in the appearance of colours but has some other origin. See also Allen (2011: sect. 4). Note that the argument of the present paper does not obviously stand or fall with the claim that all shades of colour are composed by 1-4 of the elementary attributes of the Natural Colour System. If all shades of colour are thus composed, then almost all shades of colour are visually complex. But shades of colours - most or all may turn out to be visually complex in some way other than this one. 


\section{The Remaining Colours May Also Be Visually Complex}

Now, the above case still leaves us with the six elementary attributes and the six shades — the "elementary colours" - that are composed by exactly one elementary attribute. ${ }^{1}$ I do not have nearly as strong a case to make that these are visually complex. In the case of nonelementary colours, like 3020-Y50R, I can offer a detailed account — with the help of phenomenological colour models - of what their visually accessible parts or aspects are. In the case of the elementary attributes I do not have any such account to offer: I cannot tell you what the visually accessible parts or aspects of yellowness or whiteness are, if they have any, at least not in detail and with the assurance I am arguably entitled to in the case of 3020-Y50R. However, I believe there is some reason to suppose that the elementary attributes are in fact visually complex, even if nobody today is well-equipped to state what their visually accessible components are.

I will first offer three observations in support of the claim that for all we presently know the elementary attributes may be visually complex in the present sense. I shall then offer some more tentative reasons to suppose that this is the case.

First, we have from the preceding some reason to think that we are prone to a "simplicity illusion" in the case of colours. As noted in the introduction, it is often supposed that colours are generally noncomplex or at least visually noncomplex. However, if the above account of the colours is correct this noncomplexity view embodies an illusion, at least in the case of almost all shades of colour.

Second, the history of science and thought provides some evidence that ignorance breeds distortion and error. To be ignorant is to fail to know some truth. That is not by itself to believe anything that is false or to make any other error. But there are indications, I think, that the more

\footnotetext{
${ }^{1}$ Elementary attributes are not the same as elementary colours. The elementary attribute redness is a component of all shades of red, all shades of orange, all shades of purple, and even shades of blue and shades of yellow that have a tiny bit of red in them. The elementary colour pure red, on the other hand, is not a component of any of these shades. It is a distinct shade that is composed by redness and no other elementary attribute. However, in what follows I will assume that the elementary attributes are visually complex if and only if the elementary colours are. Naturally, someone may wish to scrutinise this assumption.
} 
ignorant we are in a given area the more prone we are to false beliefs and other errors - like overconfidence or underconfidence in some belief - in that area. Consider for example Descartes' claim that a mere machine could not duplicate our abilities to engage in meaningful linguistic interactions (Descartes 1985 [1637]:56-7). ${ }^{1}$ Arguably, we still do not understand enough about this topic to say with certainty that Descartes was wrong about this. But from our present point of view, it seems that he was on this point at least overconfident. And it is arguable that an important source of this overconfidence was ignorance on Descartes' part of certain developments later to be made; in particular, the development of advanced computational machines and the understanding of how they work. From our point of view, it seems possible that a certain arrangement of the physical world should suffice to realise a certain computational system and that realising this computational system in turn suffices to duplicate our abilities to engage in meaningful linguistic interactions. At any rate, it is not so clear that this is impossible. To the extent that this seemed clearly impossible to Descartes it is plausible that this was because he was ignorant, in part about certain technological possibilities and in part about a computational level of analysis, which is theoretically intermediate between basic physical theory and observable linguistic facts and which provides illumination about how the relevant technological devices work and perhaps also illumination of aspects of our cognition.

Third, there is what we may call "the power of tacit knowledge" exemplified by, among other things, our mastery of grammar. In producing sentences, comprehending sentences and sorting sentences into grammatical and ungrammatical, we display a mastery of a grammar that appears to have the form of a complex system of rules that we can articulate only with great difficulty.

With these three observations in mind, consider the following hypothesis about the six elementary colour attributes. These attributes are in fact visually complex, and we display, in perceiving them, identifying them and discriminating them, a practical sensitivity to their visual complexity just as we exercise a practical sensitivity to blackness, whiteness,

\footnotetext{
${ }^{1}$ The remainder of this paragraph draws substantially on Stoljar (2006: sect. 7.3), which offers a much richer analysis of Descartes' claim than my discussion here. See also Stoljar (2005: sect. 5).
} 
redness and yellowness when we perceive 3020-Y50R, identify it as a certain greyish-orange and discriminate it from other shades. However, in the case of the elementary attributes we are currently largely or completely unable to articulate the complex visual characteristics that we are sensitive to. Perhaps further research will enable us to improve our theoretical understanding — in the way that the Natural Color System has improved our ability to articulate the complex visual characteristics of 3020-Y50R. In any case, our current theoretical ignorance breeds a "simplicity illusion", an erroneous disposition to regard the elementary attributes as visually noncomplex.

It is hard to see, for me, that we are justified in ruling this hypothesis out. For all we currently know things may be so.

But do we have any positive reasons to suppose that things are so? Perhaps some. Let me offer some tentative thoughts.

Perceived surface colours are (I gather) relatively well correlated with surface spectral reflectances: the proportions of light that surfaces reflect at each wavelength of the visible spectrum (approximately 400700 nanometres). ${ }^{1}$ On some accounts this correlation is evidence of identity between perceived surface colour and surface spectral reflectance (see for example Hilbert 1987, and Byrne and Hilbert 2003). ${ }^{2}$

Suppose every shade of surface colour - including every elementary colour - is identical with a certain spectral reflectance. It may seem clear that, while the elementary colours are on this supposition complex properties they are not on this supposition visually complex in the present sense. The supposition is that the distinguishable parts of aspects of an elementary colour have this character: reflecting such-and-such percentage of light and such-and-such wavelength. If these parts or aspects are visually accessible at all, they are not so in the present sense:

\footnotetext{
${ }^{1}$ Note that spectral reflectance is an illumination-independent property: the proportions of light reflected by a surface at different wavelengths is typically constant over various illuminations. Perceived surface colour is also relatively illuminationindependent: surfaces look to have the same colour under a wide variety of illuminations.

${ }^{2}$ In what follows I will focus almost entirely on surface colours. See Byrne and Hilbert (2003: sect. 3.1.2) for proposals about how the identity hypothesis concerning surface colours can be generalised into an identity hypothesis concerning the colours of surfaces, lights, filters and volumes.
} 
to see a surface as purely white, for example, is not to see it as having such parts or aspects. So it may seem.

However, things may not be so clear. I wish to suggest that at some level of grain or other spectral reflectances may be, in the present sense, visually accessible aspects of surface colours. It is clear that: to see a given surface as having a given colour is not to see it as reflecting, say, $78 \%$ of the incident light at the wavelength 412 nanometres. But there is reason to take seriously the idea that it is to see it as having a certain more coarsely individuated spectral reflectance. ${ }^{1}$

Consider a pile of sugar and piece of asphalt. One of these surfaces reflects a high and even proportion of light across the visible spectrum. The other reflects a low and even proportion of light across the visible spectrum. Most readers will know from reading about it which of these surfaces does what. But it is hardly plausible that this knowledge derives only from such "book learning". To quote from Justin Broackes:

White things reflect a fair amount of light incident upon them; black things do not. And this is not just a piece of recherché scientific fact: the behaviour is distinctive and influences our identification of the colours - it shows up in familiar things which white things do, and which people can do with them. (a) Matt-white things, since they reflect nearly all incident light, have a varied appearance highly sensitive to the variations in that light: shadows cast by other things show up clearly on a white surface. Matt-black things, by contrast, reflect little incident light; hence it makes little difference to their appearance whether shadows are falling upon them or not: little light is reflected anyway. (b) A similar fact is that it is easier to see in a room with only a weak source of light if the walls are white or pale than if they are black. (c) Water-colour painting works well on white paper, and not on black: a partly transparent wash makes quite a difference to the appearance of white paper, but practically none to the appearance of the black, which reflects little light regardless of the wash. (To change the appearance of the black, one needs to cover it, not just tinge the light being reflected by the paper behind....). (Broackes 2007:167)

\footnotetext{
${ }^{1}$ The following has been inspired by Broackes $(2003,2007)$ and Westphal (1986, $1987,1991)$ but I will not try to specify exactly how my suggestions agree and disagree with theirs.
} 
One might suggest that, while we may — tacitly or explicitly — know the facts about white and black that Broackes cite here, all this knowledge consists in or derives from learned associations. We have learned by observing regularities that in fact shadows are cast more clearly on white surfaces than on black surfaces and that water-colour painting works well on white paper. And insofar as we know about the spectral reflectances of white and black surfaces on the basis of our ordinary visual acquaintance with them, this knowledge is based on an inference to the best explanation of these observed regularities. But nothing in the visual characteristics of white or black suggest their reflectances, or the regularities; white and black are given to us in experience as two altogether simple qualities. ${ }^{1}$

However, this proposal does not seem, to me, entirely plausible and certainly not clearly right. It does not seem clearly right that we know that white reflects a high and even proportion of light in the visible spectrum only on the basis of what we have read and observed regularities concerning shadows and water-colour painting and the like. Relatedly, shifting our attention momentarily from surfaces to light sources, it does not seem clearly right that it is merely on such bases that we know that a light cannot be black (while it can be white, red, yellow, blue or green; compare Broackes 2007:168-9).

Another hypothesis, which I wish to promote for consideration, is that to see a surface as white is to see it as reflecting a certain high and even

\footnotetext{
${ }^{1}$ Locke might have approved of this suggestion. Locke urged that we clearly distinguish the ideas in our minds from the causes that produce them. The ideas of black and white are, says Locke, equally "positive" and the fact that one of them is caused by a "privation" of light is something given by an inquiry that does not belong to "the Idea, as it is in the Understanding": "Thus the Idea of Heat and Cold, Light and Darkness, White and Black, Motion and Rest, are equally clear and positive Ideas in the mind; though, perhaps, some of the causes which produce them, are barely privations in those Subjects, from whence our Senses derive those Ideas. These the Understanding, in its view of them, considers all as distinct positive Ideas, without taking notice of the Causes that produce them: which is an enquiry not belonging to the Idea, as it is in the Understanding; but the nature of the things existing without us. These are two very different things, and carefully to be distinguished; it being one thing to perceive, and know the Idea of White or Black, and quite another to examine what kind of particles they must be, and how ranged in the Superficies, to make any Object appear White or Black" (Locke 1975 [1689]: sect. 2.8.2; see also the sections that immediately follow this one).
} 
proportion of incident light, just as seeing a surface as 3020-Y50R is to see it as composed by certain proportions of blackness, whiteness, yellowness and redness. Similarly, to see a surface as black is to see it as reflecting a low and even proportion of incident light in the visible spectrum.

The difference between the two hypotheses can be appreciated - and their respective plausibility perhaps to some extent assessed - by considering a Mary-style scenario (compare Jackson 1982, 1986). Suppose someone had been confined to only ever experiencing an evenly and dimly lit scene displaying only matt surfaces in a uniform shade of grey, having had no experiences shadows and no direct experiences of light sources. Suppose now that this person were shown a black surface and a white surface, and, her visual machinery having not atrophied from her stimulus deprivation, experienced the former as black and the latter as white. Our first hypothesis above - the learned association hypothesis - would seem to predict that this subject would have no way of telling on the basis of her visual experiences which of the two surfaces reflects the highest proportion of light. There would be two novel simple qualities, each resembling grey to a certain extent but neither suggesting by its visual characteristic anything about its reflectance properties. The latter hypothesis predicts otherwise: provided that the person really sees white and black she would have visually accessible evidence about which surface reflects the highest proportion of light. My sense is that the latter prediction is the correct one.

I have so far developed my suggestion (to some extent) only for the elementary colours white and black. The remaining part of the suggestion is that to see it a surface as pure red, pure yellow, pure green or pure blue is to see it as reflecting certain uneven proportions of light in

\footnotetext{
${ }^{1}$ On the latter hypothesis, our knowledge of regularities concerning, e.g., shadows and white and black surfaces might still be in part learned associations. But they may also be suggested by the intrinsic visual characteristics of white and black surfaces. One may note that the present line of argument offers a way of combining (a) the view that surface colours are identical with surface spectral reflectances with (b) the view that colours have no aspects that are hidden to normal perceptions but are fully "laid bare" in such perceptions. (a) and (b) may both be correct if something like the present suggestion is right and colours are identical with spectral reflectances at a certain coarse level of grain.
} 
the visible spectrum. I will not develop this part, but only offer two brief remarks to indicate why I think we should take it seriously.

First, I have tried to gradually "chip away" at the sense that colours are visually simple, beginning with nonelementary colours and then moving on to the elementary colours white and black. If the project has been successful so far, that provides some (inductive) reason to believe that it can be successfully extended.

Second, there are, I again gather, asymmetries between redness, yellowness, greenness and blueness - specifically, asymmetries concerning lightness - that provide initial handles for an attempt to extend the present project to these. For example, Hård and Svedmyr (1995: 101) and Hård et al. (1996:208) record that the colours 1070-Y (10\% blackness, $20 \%$ whiteness and 70\% yellowness) and 1070-B (10\% blackness, $20 \%$ whiteness and 70\% blueness) have different "lightness values": 0.80 and 0.45 respectively. "Lightness value" is here determined by the contrast — or "border clarity" (Swedish: "gränstydlighet") — that a colour marks with shades of grey. A colour with a lightness value of 0.80 is such that, among samples of grey, it has its "minimal border clarity" with the shade of grey 2000 , composed by $20 \%$ blackness and $80 \%$ whiteness. A colour with a lightness value 0.45 has its minimal border clarity with the shades of grey 5000 and 5500, composed by 50 and $55 \%$ blackness and $50 \%$ and $45 \%$ whiteness respectively. Since 1070-Y and 1070-B contain the same amounts of blackness and whiteness, their difference in "lightness value" is apparently due to their chromatic components. Thus, yellowness and blueness appear to contribute, all by themselves and in nonequivalent ways, to a certain kind of lightness of a colour. More generally, each chromatic attribute - redness, greenness, yellowness and blueness - appears to make its own distinctive contribution to the lightness values of colours (see Hård and Svedmyr 1995: 102; Hård et al. 1996:209; see also Broackes 2007:sect. $3)$.

Admittedly, it is not clear to us that when we see a surface as having an elementary colour we see it as having a certain (relatively coarsely individuated) spectral reflectance. But then again, to those who are unfamiliar with phenomenological colour models it is perhaps not so clear that — as there is good reason to suppose — to see a surface as 3020$\mathrm{Y} 50 \mathrm{R}$ is to see it as having a certain visually complex composition. By 
the present hypothesis, our current lack of appreciation about what we see when we see as surface as white or red is rooted in ignorance. We lack a theoretical model that articulates what we see when we see white in the way that phenomenological colour models arguably articulate what we see when we see a surface as 3020-Y50R.

\section{References}

Allen, K. (2011). "Revelation and the Nature of Colour", Dialectica, 65: 153-176.

Broackes, J. (1992). "The Autonomy of Colour", in D. Charles and K.

Lennon (eds.), Reduction, Explanation and Realism, Oxford: Clarendon Press, 421-465.

Broackes, J. (2007). "Black and White and the Inverted Spectrum", Philosophical Quarterly, 57:161-175.

Byrne, A., and Hilbert, D. (2003). "Color Realism and Color Science", Behavioral and Brain Sciences, 26:3-64.

Byrne, A., and Hilbert, D. (2008). "Basic Sensible Qualities and the Structure of Appearance", Philosophical Issues, 18:385-405.

Descartes, R. (1985 [1637]). Discourse on the Method, in The Philosophical Writings of Descartes, vol. 1, trans. J. Cottingham, R. Stoothoff, and D. Murdock, Cambridge: Cambridge University Press.

Hering, E. (1964 [1920]). Outlines of a Theory of the Light Sense, trans. L. Hurvich and D. Jameson, Cambridge, MA: Harvard University Press.

Hilbert, D. (1987). Color and Color Perception: A Study in Anthropocentric Realism, Stanford: Center for the Study of Language and Information, Stanford University.

Hume, D. (1978 [1739]). A Treatise of Human Nature, P. Nidditch (ed.), Oxford: Oxford University Press.

Hård, A., and Sivik, L. (1981). "NCS - Natural Color System: A Swedish Standard for Color Notation", Color Research \& Application, 6: 129-138.

Hård, A., Sivik, L., and Tonnquist, G. (1996). "NCS, Natural Color System - From concept to Research and Applications, parts 1 \& 2", Color Research \& Application, 21:180-220. 
Hård, A., and Svedmyr, Å. (1995). Färgsystemet NCS, Stockholm: Byggforskningsrådet.

Jackson, F. (1982). "Epiphenomenal Qualia", Philosophical Quarterly, 32: $127-136$.

Jackson, F. (1986). "What Mary Didn't Know", Journal of Philosophy, 83:291-5.

Locke, J. (1975 [1689]). An Essay Concerning Human Understanding, P. Nidditch (ed.), Oxford: Oxford University Press.

Mizrahi, V. (2009). "Is Colour Composition Phenomenal?", in D. Skusevich and P. Matikas (eds.), Color Perception: Physiology, Processes and Analysis, Nova Science Publishers. Online draft at http://www.mizrahi.ch/philo/Composition_mizrahi.pdf; accessed November 9, 2012.

Pridmore, R. (2006). "14th Century Example of the Four Unique Hues", Color Research \& Application, 31:364-365.

Saunders, B. A. C., and van Brakel, J. (1997). "Are There Nontrivial Constraints on Colour Categorization?", Behavioral and Brain Sciences, 20: 167-179.

Stoljar, D. (2005). "Physicalism and Phenomenal Concepts", Mind and Language, 20:469-494.

Stoljar, D. (2006). Ignorance and Imagination. The Epistemic Origin of the Problem of Consciousness, Oxford: Oxford University Press.

Sundström, P. (2008). "Humes utelämnade nyans av blått”, Filosofisk Tidskrift, 29:18-33.

Thomas, N. (2012). "Mental Imagery", in E. Zalta (ed.), The Stanford Encyclopedia of Philosophy, winter 2012, http://plato.stanford.edu/archives/win2012/entries/mental-imagery/

Westphal, J. (1986). "White", Mind, 95:310-28.

Westphal, J. (1989). "Black", Mind, 98:585-9.

Westphal, J. (1991). Colour. A Philosophical Introduction, Oxford: Blackwell. 